\title{
Efficiency Loss in HPGe Detectors due to Beta and Gamma Sum Coincidence
}

\author{
Ruy M. Castro, \\ Divisão de Sensoriamento Remoto, Instituto de Estudos Avançados, \\ Centro Técnico Aeroespacial, Rodovia dos Tamoios Km 5,5, \\ Torrão de Ouro, Cep 12228-840, São José dos Campos, SP, Brazil \\ Vito R. Vanin, Nora L. Maidana, Paulo R. Pascholati, \\ Laboratório do Acelerador Linear, Instituto de Física, Universidade de São Paulo, \\ Travessa R 187, Cidade Universitária, CEP: 05508-900, São Paulo, SP, Brazil \\ Mauro S. Dias, and Marina F. Koskinas \\ Laboratório de Metrologia Nuclear, Instituto de Pesquisas Energéticas e Nucleares, \\ Travessa R 400,Cidade Universitária, CEP: 05508-900, São Paulo, SP , Brazil
}

Received on 8 June, 2005

\begin{abstract}
Among the secondary detection effects in gamma-ray spectroscopy with HPGe detectors that reduce the peak area and must be properly taken into account in accurate measurements, the less well known is counting loss by beta and gamma-ray sum coincidence. The fraction of lost counts was estimated assuming that a photon detection event can sum either with the coincident beta-ray or its bremsstrahlung in the detector capsule or surrounding materials. The beta-ray was tracked through all the materials between source and detector. The result was calculated as a sequence of integral expressions evaluated numerically, without resorting to simulation. It is shown that the counting losses due to this effect are negligible for most calibration sources in typical detector arrangements.
\end{abstract}

\section{INTRODUCTION}

Quantitative gamma-ray spectroscopy of radioactive sources with Hyper-Pure Germanium (HPGe) detectors is affected by many secondary detection effects. This paper deals with the counting losses due to coincidental detection of a gammaray and the electron feeding the excited level. Not much attention has been paid to this effect, usually assumed to be insignificant, without a detailed explanation in the few references available.

The counting loss occurs when the electron, after traversing the materials between source and detector, enters the HPGe active region, adding some charge to a simultaneous photon detection event, displacing it from the expected energy value. Another possibility of loss is due to the detection of bremsstrahlung photons generated while the electron follows its path.

A procedure to estimate these sum losses is presented here, in function of the source properties and detector characteristics: beta-energy endpoint; intermediate levels life times; detector size, and Ge dead-layer thickness. The importance for many common calibration sources in a typical source-detector arrangement is shown.

*Also at: Laboratório do Acelerador Linear, Instituto de Física, Universidade de São Paulo, Travessa R 187, Cidade Universitária, CEP: 05508-900, São Paulo, SP, Brazil; and Departamento de Matemática e Física, Universidade de Taubaté, Rua Daniel Danelli, s/n, Cep: 12060-440, Taubaté, SP, Brazil

\section{COUNTING LOSS}

Consider the decay scheme shown in Figure 1, where prompt gamma-rays follow beta transitions. The observed full energy peak area of a gamma-ray transition, already corrected for beta-gamma sum losses, is given by

$$
\operatorname{Area}_{\gamma}=\frac{\Omega \cdot \Delta t \cdot \varepsilon\left(E_{\gamma}\right) \cdot P \gamma}{1+\alpha_{\gamma}} \cdot\left(1-f_{\beta} \cdot \varepsilon_{\beta}\right)
$$

where:

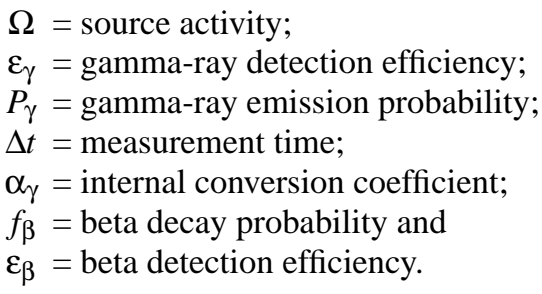

A fraction of the events, given by $f_{\beta} \cdot \varepsilon_{\beta}$ in the second term in parentheses in the formula above, corresponds to the correction for beta-gamma sum losses, where the beta detection efficiency, $\varepsilon_{\beta}$, takes into account the geometric efficiency $\left(\varepsilon_{g e o \beta}\right)$, combined with the probabilities that a beta $\left(P_{\beta}\right)$ or an electron bremsstrahlung photon $\left(P_{\text {Brems }}\right)$ reach the detector:

$$
\varepsilon_{\beta}=\varepsilon_{\text {geo } \beta} \cdot\left(P_{\beta}+P_{\text {Brems }}\right)
$$

with the factors entering this formula calculated as follows. The geometric efficiency is estimated from the source to detector distance and the detector area; the probability of the electron reaching the detector active region is determined by 


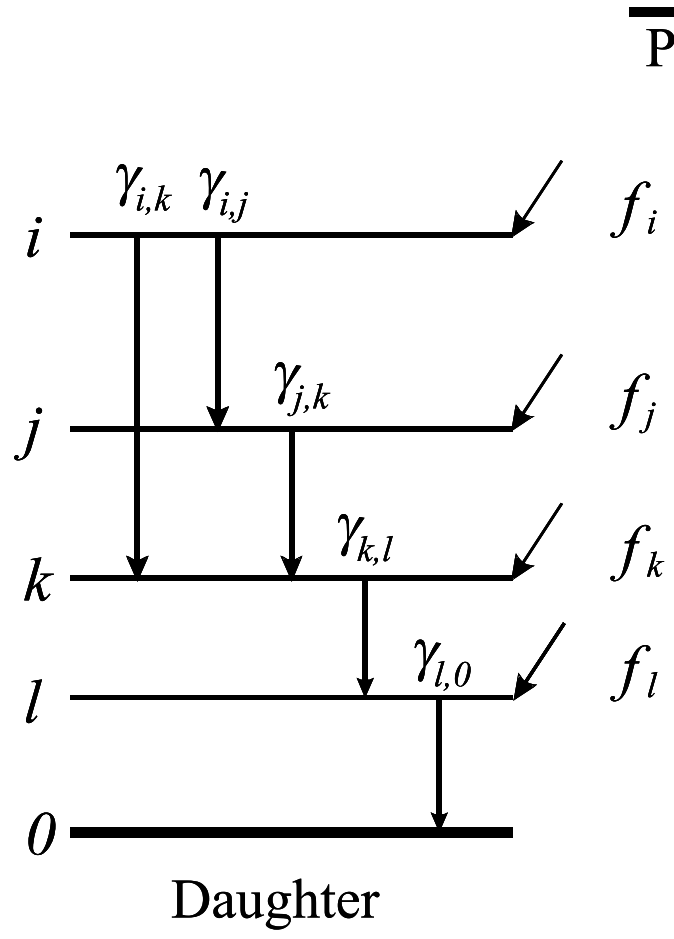

FIG. 1: Generic beta decay scheme.

the transmission probability through the materials in the electron path, including the detector cap and the Ge dead layer; the probability of a electron Bremsstrahlung reaching the detector active region was calculated as the rate of photon production by a beta hitting the dead layer of the HPGe detector times the transmission probability along the electron trajectory.

\section{A. Beta-gamma sum}

This effect will happen whenever a photon is detected together with one electron has enough energy to reach the detector active volume. Since any amount of energy deposited by the electron in the detector simultaneously with the detection of a photon will lead to the loss of the event, $P_{\beta}$ will depend on the electron energy threshold to reach the detector, and is given by the integral of the beta spectrum between this threshold and the beta endpoint energy $\left(E_{\beta \max }\right)$. The electron energy threshold to enter the detector is calculated from the energy loss coefficient in the materials $(\mu)$, and the material thickness $(x)$ :

$$
E_{\beta}=E_{\beta_{\max }}-\mu\left(E_{\beta}, x\right)
$$

For a typical setup, with a radioactive source hold in the normal atmosphere in front of a detector, there are just a few materials involved. The energy loss coefficients for these materials can be seen in Figure 2 .

The beta decay spectra was calculated by means of the model of Konopinsk [2] for allowed transitions, see Figure 3. A correction for electron reflection in thick materials [3-5]

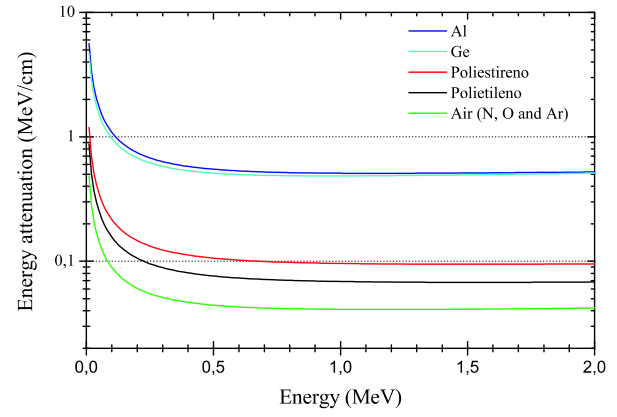

FIG. 2: Energy loss coefficient in common materials in gamma-ray spectroscopy arrangements [1] as function of the beta energy.

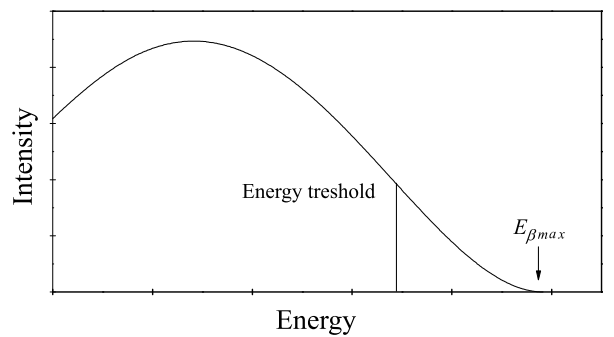

FIG. 3: Beta decay spectrum for allowed transitions, showing the electron energy threshold to reach the detector active region and the beta endpoint energy $\left(E_{\beta \max }\right)$.

was applied to the value obtained integrating the beta spectra above the energy threshold.

\section{B. Sum with electron Bremsstrahlung}

Since high-energy electrons are more likely to produce bremsstrahlung, some care must be taken to avoid double counting of the losses. Also, the production of bremsstrahlung in the low $\mathrm{Z}$ materials forming the source holder and the atmosphere is negligible compared to that in the relatively high $\mathrm{Z}$ of the Ge dead layer. Hence, $P_{\text {Brems }}$ corresponds to the sum with bremsstrahlung produced by the electrons with energies between that necessary to reach the detector dead layer and the threshold energy $E_{\beta}$. The rate of bremsstrahlung photon production was obtained by interpolation of the table compiled by Seltzer [6]. Figure 4 shows the distribution of bremsstrahlung photons. As most of the electrons have low energy, the production of bremsstrahlung is small and concentrated in the low energy end of the photon spectrum.

\section{APPLICATION}

This method was applied to estimate the counting losses due to beta and gamma-ray sum coincidence for some usual standard sources assuming a typical HPGe detector-source arrangement, shown schematically in Figure 5. 


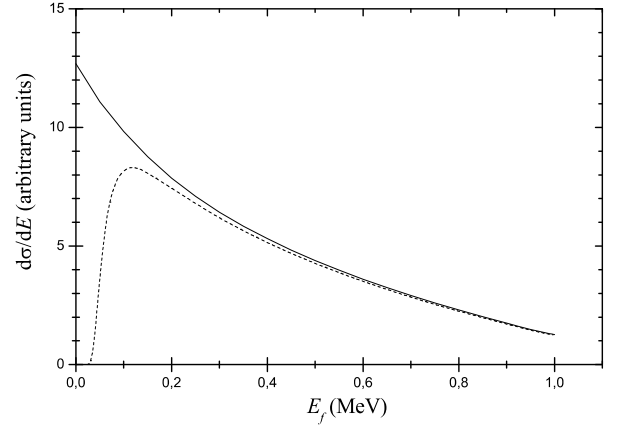

FIG. 4: Distribution of bremsstrahlung photons [6] produced by 1 $\mathrm{MeV}$ electrons. The dashed line takes into account the absorption in the dead layer of the detector.

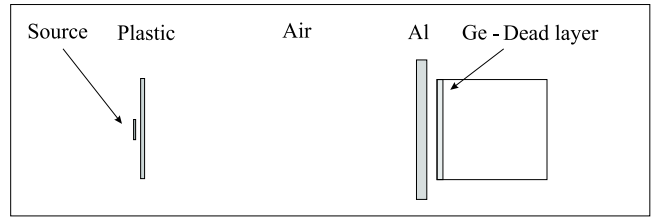

FIG. 5: Typical detection arrangement. The source [7, 8] has a $0.40 \mathrm{~mm}$ of polyethylene, the detector [9] has a $1.27 \mathrm{~mm} \mathrm{Al}$ cover and a $0.70 \mathrm{~mm}$ of Ge dead layer and they are separated by $20 \mathrm{~cm}$ of air.

\section{A. Beta decay of some calibration sources}

Since the usual source mounting is made of low $\mathrm{Z}$ material and is thin [7-10], then the electron threshold energy to reach the detector does not depend significantly on the particular source and was estimated in about $1.3 \mathrm{MeV}$.

After checking the requirements for beta and gamma sum (beta endpoint energy greater than the electron threshold energy and half-lives of intermediate levels involved in the decay) we found that, among the following sources used frequently in detector calibration: ${ }^{46} \mathrm{Sc} ;{ }^{60} \mathrm{Co} ;{ }^{94} \mathrm{Nb} ;{ }^{137} \mathrm{Cs}$; ${ }^{203} \mathrm{Hg} ;{ }^{22} \mathrm{Na} ;{ }^{58} \mathrm{Co} ;{ }^{95} \mathrm{Nb} ;{ }^{141} \mathrm{Ce}$ and ${ }^{152} \mathrm{Eu}$, there are only 2 cases where the sum is possible.

One of the cases happens in the decay of the ${ }^{60} \mathrm{Co}$, where the $1332 \mathrm{keV}$ level is fed by $\beta^{-}$with endpoint energy of $1492 \mathrm{keV}$ with probability of $0.06 \%$. The other case is the $1474 \mathrm{keV} \beta^{-}$decay of the ${ }^{152} \mathrm{Eu}$ to $344 \mathrm{keV}$ level of the ${ }^{152} \mathrm{Gd}$ with $8,2 \%$ probability. Although these beta decays are 1st and 2 nd forbidden, the spectrum shape of the allowed transition [2] can be used by for an order of magnitude estimate.

\section{B. Estimation of counting losses}

The arrangement shown in Figure 5 has a geometric efficiency of $0.54 \%$. The probabilities of the detection of betas $\left(P_{\beta}\right)$ are: $1.0 \%$ and $0.7 \%$ in the cases of ${ }^{152} \mathrm{Eu}$ and ${ }^{60} \mathrm{Co}$, respectively, while the probability of detection of the bremsstrahlung photon, $\left(P_{\text {Brems }}\right)$, is about $0.9 \%$ for both cases.

Therefore, the correction $\left(f_{\beta} \cdot \varepsilon_{\beta}\right)$ can be estimated in about $7 \cdot 10^{-6}$ and $1.2 \cdot 10^{-7}$, of the peak areas of the $\gamma 344 \mathrm{keV}$ following ${ }^{152} \mathrm{Eu}$ decay and the $\gamma 1332 \mathrm{keV}$ following the ${ }^{60} \mathrm{Co}$ decay, respectively. Thus, the corrections for these cases are negligible.

\section{CONCLUSION}

The effect of beta and gamma ray sum will be significant when using HPGe detectors with $\mathrm{Al}$ capsule only for radioactive sources where beta transitions have high endpoint energies, well above $1.3 \mathrm{MeV}$, and feed excited levels of the daughter nucleus. Therefore, it will rarely manifest in usual residual radioactive source measurements, and in none of the common calibration sources that we checked.

\section{Acknowledgments}

We wish to acknowledge the CNPq, FAPESP and AIEA for the partial financial support.
[1] L. Pages, E. Bertel, H. Joffre, and L. Sklavenitis, Energy Loss, Range, and Bremsstrahlung Yield for $10 \mathrm{keV}$ to $100 \mathrm{MeV}$ Electrons in Various Elements and Chemical Compounds, Atomic Data, 4, 1 (1972)

[2] E. Konopinski, The Theory of Beta Radioactivity, Oxford, 1966

[3] G. F. Knoll, Radiation Detection and Mesurement John Wiley \& Sons, $2^{a}$ Ed., 1989

[4] T. Tabata, R. Ito, and S. Okabe, An Empirical Equation for the Backscattering Coefficient of Electrons, Nucl. Inst. and Meth., 94, 509 (1971).

[5] Y. Zhou, Z. An, Q. Tang and C. Tang, Reflection of Electrons from Material Surfaces, Radiation Physics and Chemistry, 57, $731(2000)$
[6] S. M. Seltzer and M. J. Berger, Bremsstrahlung Spectra From Electrons Interactions with Screened Atomic Nuclei and Orbital electrons, Nucl. Inst. and Meth. in Phys. Res. B 12, 95 (1985).

[7] M. F. Koskinas, Características de fontes - Private communication, IPEN, (1997)

[8] Amersham, Gamma Reference Source Set, Data Sheet 11188, (1981)

[9] Ortec, Quality assurance data Sheet, detector Model No GEM30185-P, Serial No 34-TP30930A

[10] R. M Castro, Transformando o ${ }^{152} \mathrm{Eu}$ em um Padrão de Calibração de Eficiência para Deteção de Gamas, Phd. Thesis, IFUSP (2003) 